\title{
Universality vs experience: A cross-cultural pilot study on the consonance effect in music at different altitudes
}

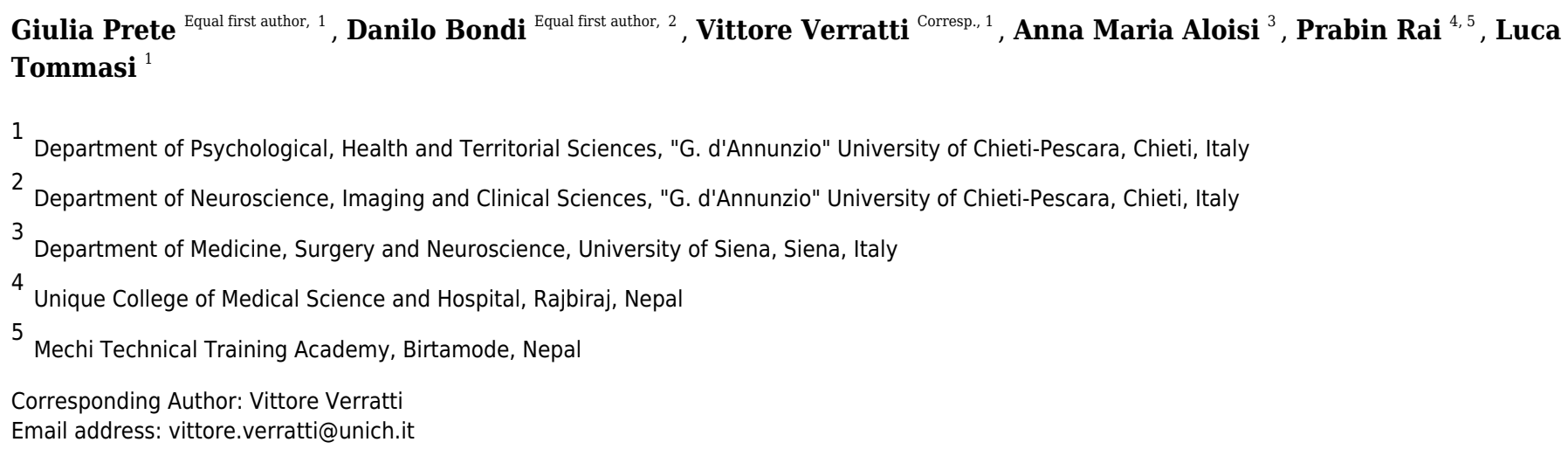

Background. Previous studies have shown that music preferences are influenced by cultural "rules", some others have suggested a universal preference for some features over others. Methods. We investigated cultural differences on the "consonance effect", consisting in higher pleasantness judgments for consonant compared to dissonant chords according to the Western definition of music: Italian and Himalayan participants were asked to express pleasantness judgments for consonant and dissonant chords. An Italian and a Nepalese sample were tested both at $1450 \mathrm{~m}$ and at $4750 \mathrm{~m}$ of altitude, with the further aim to evaluate the effect of hypoxia on this task. A third sample consisted of two subgroups of Sherpas: lowlanders (1450 m of altitude), often exposed to Western music, and highlanders (3427 m of altitude), less exposed to Western music. All Sherpas were tested where they lived. Results. Independently from the altitude, results confirmed the consonance effect in the Italian sample, and the absence of such effect in the Nepalese sample. Lowlander Sherpas revealed the consonance effect, but highlander Sherpas did not show this effect. Conclusions. Results of this pilot study show that neither hypoxia (altitude), nor demographic features (age, schooling, or playing music), nor ethnicity per se influence the consonance effect. We conclude that music preferences are attributable to music exposure. 
1 Universality vs experience: A cross-cultural pilot study on the consonance effect in music at different altitudes

3

4 Giulia Prete $^{1 *}$, Danilo Bondi ${ }^{2 *}$, Vittore Verratti ${ }^{1}$, Anna Maria Aloisi ${ }^{3}$, Prabin Rai ${ }^{4,5}$, Luca

5 Tommasi $^{1}$

6 *These authors contributed equally to the work

7

8 1Department of Psychological, Health and Territorial Sciences, "G. d'Annunzio" University of

9 Chieti-Pescara, Italy

10 2Department of Neuroscience, Imaging and Clinical Sciences, "G. d'Annunzio" University of

11 Chieti-Pescara, Italy

$12{ }^{3}$ Department of Medicine, Surgery and Neuroscience, University of Siena, Italy

$13{ }^{4}$ Unique College of Medical Science and Hospital, Rajbiraj, Nepal

$14 \quad{ }^{5}$ Mechi Technical Training Academy, Birtamode, Nepal

15

Corresponding author

17 Correspondence to Prof. Vittore Verratti

18 vittore.verratti@unich.it

19

20 


\section{Abstract}

22

23

24

25

Background. Previous studies have shown that music preferences are influenced by cultural "rules", some others have suggested a universal preference for some features over others. Methods. We investigated cultural differences on the "consonance effect", consisting in higher pleasantness judgments for consonant compared to dissonant chords - according to the Western definition of music: Italian and Himalayan participants were asked to express pleasantness judgments for consonant and dissonant chords. An Italian and a Nepalese sample were tested both at $1450 \mathrm{~m}$ and at $4750 \mathrm{~m}$ of altitude, with the further aim to evaluate the effect of hypoxia on this task. A third sample consisted of two subgroups of Sherpas: lowlanders (1450 $\mathrm{m}$ of altitude), often exposed to Western music, and highlanders (3427 m of altitude), less exposed to Western music. All Sherpas were tested where they lived. Results. Independently from the altitude, results confirmed the consonance effect in the Italian sample, and the absence of such effect in the Nepalese sample. Lowlander Sherpas revealed the consonance effect, but highlander Sherpas did not show this effect. Conclusions. Results of this pilot study show that neither hypoxia (altitude), nor demographic features (age, schooling, or playing music), nor ethnicity per se influence the consonance effect. We conclude that music preferences are attributable to music exposure.

Keywords: Consonance; Dissonance; Music pleasantness; Hypoxia; Culture; Altitude.

Running title: Consonance effect: more cultural than universal 


\section{Introduction}

The topic of music pleasantness extends towards a plethora of scientific fields, from psychology to neurology, from musicology to sociology, from ethnology to biology (Cross, 2003; Bowling \& Purves, 2015; Bowling et al., 2017). The first issue in this domain is the definition of music pleasantness itself, followed by the difficulty in understanding whether universal laws exist according to which music can be categorized as pleasant vs unpleasant, or it is a personal preference, possibly influenced by culture (Cazden, 1980; Higgins, 2012; Harrison $\&$ Pearce, 2020). Concerning the first issue, in Western music pleasantness seems to be linked to vertical harmonicity (Terhardt, 1984; McDermott, Lehr \& Oxenham, 2010; McDermott et al., 2016). Harmonicity, in fact, can be referred to either a "vertical" dimension, with isolated chord presentation, or to a "horizontal" dimension, with sequential chords presentation. Here the term harmonicity will be used to refer to vertical harmonicity. Harmonicity is obtained when the pitches in a chord are related by simple integer ratios of frequencies and in Western culture it would contribute to the perceived consonance and to the following pleasantness of the chord. We can summarize this concept in the following way: a harmonic chord (a set of notes played together) sounds as pleasant if it is consonant (Bowling \& Purves, 2015). In Western culture, in fact, consonance is associated with pleasantness, stability and relaxation, and dissonance is associated with unpleasantness (the so-called "consonance effect"; Prete et al., 2015, 2019).

A chord is obtained from the overlapping of more pure tones defined as partials. When perceived together, neighboring partials could lead to interference, producing dissonance. Interference is obtained when the spectral components in a chord are close enough to elicit masking and beating, which have been proposed to be the acoustic base for perceived roughness (for a deeper dissertation on different theories about consonance see Tramo et al., 2001; Harrison 
$65 \&$ Pearce, 2020). These principles of music consonance seem to be culturally defined, in fact

66 they are valid in Western music, which is based on harmonicity. Differently from Western

67 listeners, who perceive interference as unpleasant, in some non-Western cultures beating is

68 associated to consonance (e.g., Messner, 1981), showing that the aesthetic appreciation of

69 interference is culturally defined. According to this view, music harmonicity is a central feature

70 of Western music, but it is less diffuse or absent in other cultures (Malm, 1996). This leads to the

71 second issue raised above: if harmonicity is the base for the consonance effect in Western music,

72 how are consonant and dissonant chords evaluated in those cultures in which harmonicity is not

73 present? Answering this question can help in defining music preferences as established by nature

74 or by nurture (Bowling et al., 2017): on the one hand, some agreement should be expected in the

75 evaluation of pleasant vs unpleasant music across different cultures if music preferences are

76 innate and biologically determined, but - on the other hand - different preferences for different

77 music should be found among cultures if music preference is culturally defined.

78 Both of the aforementioned hypotheses found support in previous studies. Some reports

79 suggested music consonance to be a universal principle, independent from the cultural

80 environment: for instance, a preference for consonance was described even in different species,

81 such as birds and monkeys (Fishman et al., 2001; Chiandetti \& Vallortigara, 2011), and it has

82 been shown that 2-month-old infants prefer to listen to consonant over dissonant chords (Trainor,

83 Tsang \& Cheung, 2002). Nevertheless, this evidence was not confirmed in a different study with

84 6-month-old infants (Plantinga \& Trehub, 2014), and in other studies with monkeys (McDermott

$85 \&$ Hauser, 2004), suggesting that music consonance might not be universal, developing instead

86 with experience and listening habits, namely on culture (McDermott et al., 2016). Along similar 
87 lines, it has been suggested that the consonance/dissonance spectrum would be context-sensitive

88 to the original ecological listening experience (Popescu et al., 2019).

89

90

91

92

93

94

95

96

97

98

A possible way to empirically disentangle the innate vs cultural origin of music preferences is represented by cross-cultural studies: the comparison among music preferences expressed by individuals belonging to different cultures can help in shedding light on this topic. In this respect, McDermott and colleagues (McDermott et al., 2016) provided evidence for a cultural basis of music preferences, showing that music pleasantness is attributable to exposure to musical harmonicity. In particular, they tested Western (US Americans) participants, native Amazonian participants without exposure to Western culture (Tsimane'), and Bolivians living either in the capital city or in a rural town. The results showed that the Tsimane' did not evaluate differently consonant and dissonant chords, the participants from US confirmed the expected consonance effect, whereas the Bolivian groups were placed in the middle, showing a "consonance effect", but less strong than that of US participants. These findings are in accordance with previous studies in which different populations have been tested. For instance, Maher (1976) found different "restful/restless" ratings in Canadians and Indians for harmonic intervals, even if Butler and Daston (1968) found no difference in dyadic chords preference between Americans and Japanese, leading the authors to conclude that music preference could be dissociated with respect to the perception of consonance. All of these results confirm the usefulness of cross-cultural studies in the investigation of the possible biological substrate of music preferences.

With the present multicultural and globalized world, it is increasingly difficult to explore genuine cross-cultural variations (Segall et al., 1990). English language-based elements are indeed a key factor of modern cultural evenness, and this trend did not spare music listening habits. In this regard, the possibilities offered by extreme environmental research projects, such 
110 as the present "Kanchenjunga Exploration \& Physiology" project, may be really useful. Indeed,

111 the history of altitude physiology still continues to be enriched, especially by the recent efforts

112 made to understand the peculiarities of high-altitude populations (West, 2016). For these reasons,

113 in the present study we tested European and Himalayan participants, exploiting the different

114 music cultures and habits of the two samples. Nepal had its declaration of democracy only in

115 1951, thus only in the last decades it started to open to different cultures, mainly near India and

116 China (Dinnerstein \& Alter, 2018). In the same year Radio Nepal was created and it broadcasted

117 in Kathmandu alone, being the only radio station until the 1990s. Nepal has its own music

118 culture, even if it is quite heterogeneous (Henderson, 2002), also due to the fact that these

119 territories are inhabited by a mix of different populations, sometimes separated by mountains and

120 thus very different from one another, some other times living together (Greene, 2003). In

121 particular, in the North-East of the country, Tibetan groups are frequent, including Sherpas, an

122 independent ethnical group coming from Tibet. Nepalese music is a plethora of numerous

123 traditions and presently it is changing deeply in terms of content and context. Newar music

124 (representing the societies of the Kathmandu valley) is characterized by complex rhythmic cycles

125 (Prajapati, 2018). The traditional Nepalese folk music shares with North India and neighbor

126 countries the expression of tonality through melody, from an expanded set of heptatonic scales.

127 Thus, in respect to Western music, it lacks the harmonic function of chords. In the music of

128 North India and neighbor countries, tonal hierarchy is characterized by addition or deletion of

129 tones, deviating from the underlying scale (Castellano, Bharucha \& Krumhansl, 1984). Tibetan

130 music (representing the Sherpa society) is mainly based in the vowel modification and

131 contouring of tones, without the Western function of chords and with melodies consisting of

132 several melodic patterns and little melodic ranges (Tsukamoto, 1983). 
Over 100 million persons live at high altitude, throughout Asia, East Africa, and North,

134 Central, and South America (Moore, 2001): testing these population requires to detect if changes are due to chronic hypoxia exposure or typical culture. In this regard, the notable increase of

136 altitude traveling in last decades opens novel intriguing perspectives. Evaluating the effect of

137 living altitude or testing altitude may allow to focus specifically on cultural habits.

138 The "Kanchenjunga Exploration \& Physiology" project, a scientific expedition that took

139 place mainly in the Himalayas, offered us the possibility to investigate music preferences in both

140 Nepalese and Sherpa samples, and this in turns allowed us not only to compare music

141 preferences of Western (European) and Himalayan participants, but also to further investigate

142 differences between ethnical groups living close to one another (Nepalese and Sherpa), but

143 having different music habits. It is well known that altitude hypoxia negatively affects some 144 aspects of physiology and, specifically, sensory systems (Cingi, Erkan \& Rettinger, 2010; Jha, 145 2012; Ruffini et al., 2015); regarding pleasantness, little is known about the role of hypoxia: e.g. 146 no effect was found for thermal comfort (Golja et al., 2005), no effect for flavors pleasantness, 147 and specific effects were found for the evaluation of pleasantness of individual odors due to 148 hypoxia (Huppertz et al., 2018). To our knowledge, there is still a lack of results regarding the 149 effect of altitude hypoxia on preferences related to music pleasantness. As well, cognitive and 150 emotional adaptations were reported during altitude volitional exposure or during hypoxia 151 confinement, suggesting that high motivation may drive pleasantness of the experience, even 152 with highly demanding hypoxic exposure (Karinen \& Tuomisto, 2017; Stavrou et al., 2018). In 153 addition, despite the fact that the effect of music on mood has been extensively studied, also in 154 their modulation of stress response (Koelsch et al., 2016), few studies, if any, investigated the 
155 modulation of mood in specific measures of music pleasantness. We therefore saw, among the 156 other research lines of the project, the special possibility to explore the above-mentioned topics.

157

158

159

160

161

162

\section{Aim}

This study aimed to evaluate cultural variation in music consonance/dissonance-derived pleasantness, a possible role of altitude and a possible mediation by mood, age, being a musician and Western music listening habit. To this aim, we exploited a musical test already used to describe the consonance effect in Western participants (see Prete et al., 2015), with three samples belonging to different cultures: Italians, Nepalese and Sherpas. Italian and Nepalese samples were tested both at lower and higher altitudes with the aim to evaluate the possible effect of hypoxia at high altitude. Moreover, a mood questionnaire was also administered to the Italian sample, in order to investigate the effect of hypoxia also on this variable. Starting from the results described by McDermott and colleagues (McDermott et al., 2016), we expected to confirm the consonance effect in the Italian group, and likely in lowlander Sherpas, often exposed to Western music, and we did not expect to find such effect in the other groups. Concerning altitude, due to the absence of specific evidence in this regard, we did not have specific expectations on the effect of hypoxia on music pleasantness evaluation; however, considering previous evidence about the role of hypoxia on sensorial functions and pleasantness, we may hypothesize hypoxia may affect somehow music pleasantness. Moreover, we also took into account the possible effects of time spent listening to music, frequency of exposure to Western music, years of education and playing music or otherwise on the consonance effect.

\section{Materials and methods}




\section{Participants}

The task was carried out by three groups of participants $(\mathrm{N}=22$; see Table 1 for demographic information self-reported by each participant): Italians, Nepalese, and Sherpas. The Italian group was composed of six healthy Caucasian lowlanders ( 5 males, 1 female; age: 43.83 \pm 15.30 years; BMI: $25.81 \pm 3.25 \mathrm{Kg} / \mathrm{m}^{2}$; schooling: $18.50 \pm 3.27$ years), who were used to listen to Western music, on average 1.5 hours/day. The Italian group spoke on average 1.33 foreign languages (range: 0-2, all Western languages). The Nepalese group was composed of six healthy porters (workers who carry loads during altitude expeditions), lowland dwellers (all males; age: $30.33 \pm 8.55$ years; BMI: $24.36 \pm 4.70 \mathrm{Kg} / \mathrm{m}^{2}$; schooling: $5.86 \pm 4.71$ years), who declared to listen on average 1.37 hours/day, but only one participant reported to listen to Western music sometimes. This group also reported to speak on average 1.33 foreign languages (range: $0-3$, including 3 participants who spoke English; the other spoke Eastern languages). The third group was composed by ten healthy Sherpa (all males). This group was further divided into five lowlanders (age: $28.60 \pm 5.23$ years, schooling: $11.20 \pm 3.63$ years) and five highlanders (age: $37.00 \pm 16.51$ years, schooling: $6.40 \pm 4.28$ years). Lowlander Sherpas were used to spend much time to listen music (on average, 4 hours/day) and, except one participant, they declared to be often exposed to Western music. This seems to be in line with the self-reported data according to which all of them spoke at least three foreign languages, included English (range: 3-4, average: 3.2). Highlander Sherpas listened to less music ( 0.9 hours/day) and only one participant in this subsample stated to sometimes listen to Western music. Only this participant and another one in this subsample declared to speak English, with a mean of 1.6 foreign languages spoken in the subsample (range: 0-3; Eastern languages). Among all participants, 7 of them played music since at least 3 years (2 Italian, 1 Nepalese, 2 lowlander and 2 highlander Sherpas). We decided to 
201 consider three main groups according to the participants' ethnicity (Italian, Nepalese and

202 Sherpa), independently of their reported exposure to Western music, knowledge of Western

203 languages, and the other demographic features, in order to investigate the pure effect of ethnicity

204 on the music pleasantness evaluation. Table 1 summarizes demographic data of the whole

205 sample. No participant reported symptoms of Acute Mountain Sickness (AMS) during the trek.

206

207

208

209

210

211

212

213

214

215

216

217

218

219

220

221

222

\section{Design of the study}

*** Table 1 here ***

The research project "Kanchenjunga Exploration \& Physiology” was a subset of

"Environmentally-modulated metabolic adaptation to hypoxia in altitude natives and sea-level dwellers: from integrative to molecular (proteomics, epigenetics, and ROS) level" approved by the Ethical Review Board of the Nepal Health Research Council (NHRC, reference number 458). All study procedures were performed in accordance with the ethical standards of the 1964 Helsinki declaration and its later amendments or comparable ethical standards. All participants provided their written informed consents.

The expedition consisted of a combined circuit of $300 \mathrm{Km}$ distance (south and north base camps), covering a daily average of 6 hours walk, for a total of 110 hours, along a demanding route with ascent and descent covering totally over 16000 meters in altitude, in the Himalayan mountain range of eastern Nepal, at the border with Sikkim (India). The project investigated adaptive physiological responses during a trekking at moderate and high altitude, in different experimental groups (Italians and Nepalese, for more details see Participant section). 
224 (group 2) participants at lower and higher altitudes (respectively LA and HA2, see Figure 1).

225 Italians and Nepalese carried out the same auditory test twice, in Kathmandu (1450 m) and in

226 Lhonak $(4780 \mathrm{~m})$. Only Italian participants also underwent mood state testing on the same days,

227 at the end of the auditory test. Sherpas were tested once, in a between-subjects design: lowlander

228 Sherpas were tested in Kathmandu, where they lived, whereas highlander Sherpas were tested in

229 Ghunsa (HA1, see Figure 1) at 3427 m, where they lived. A native Nepalese speaker assisted

230 during the test, both in Kathmandu, Ghunsa, and Lhonak, confirming participants understood the 231 task.

232

**Figure 1 here**

\section{Stimuli and procedure}

The stimulus set was composed of 24 triad chords, including 12 consonant and 12

dissonant chords, according to the Western music definition of consonance and dissonance. The same set of stimuli and paradigm had already been used with an Italian sample (Prete et al., 2015), confirming the "consonance effect", namely the lower and higher pleasantness evaluation of dissonant and consonant chords, respectively. Consonant chords consisted of major $3^{\text {rd }}$ intervals (interval ratio 4:5), perfect $5^{\text {th }}$ intervals (interval ratio $2: 3$ ) and minor $3^{\text {rd }}$ and $6^{\text {th }}$

242 intervals (interval ratio 5:6 and 5:8, respectively); dissonant chords consisted of minor and major $2432^{\text {nd }}$ intervals (interval ratio 15:16 and 8:9) and in minor and major $7^{\text {th }}$ intervals (interval ratio

$2449: 16$ and 8:15). By exploiting these intervals, three consonant and three dissonant triads were created with a piano timbre and, by means of GoldWave v5.25 software (GoldWave Inc., 
246 Canada): in particular, in the consonant chords all intervals among three notes were consonant (C

$\left.247-E^{b}-G ; C-E-G ; C-E-A^{b}\right)$, and in the dissonant chords all intervals among three notes

248 were dissonant $\left(B^{\#}-C^{\#}-D ; C-D^{b}-B ; C-A^{\#}-B\right)$. Then, these six chords were transposed up

249 by 4, 6 and 9 semitones, obtaining four chords for each of the original ones: the position of the

250 tonic of each chord was transposed, and the distance among notes remained the same. In this

251 way, 12 consonant and 12 dissonant chords were created (stimuli are available as Supplementary

252 materials). Each stimulus lasted $1330 \mathrm{~ms}$ and included $630 \mathrm{~ms}$ of linear fade out and was

253 repeated four times for a total set of 96 stimuli, presented in a random order by means of

254 headphones (see Figure 2).

255 The paradigm was presented by means of a tablet PC (Microsoft Surface Pro 6) and it was

256 controlled by the experimenter, who sat behind the participant for the whole task. Before the

257 beginning of the task, experimental instructions were verbally presented: participants were

258 instructed that very brief audio clips would be presented one at time, and that they will be

259 required to express a pleasantness judgment for each clip, by using a 4 point-Likert scale $(1=$ not

260 at all; $2=$ little; $3=$ enough; 4=very much). The list of four responses were also printed in the

261 mother tongue of each participant in order to avoid possible confounding effects on the scale

262 used, and to make sure that the response provided by the participant could be clearly recognized

263 by the experimenter. Once the response was provided, the experimenter recorded the number

264 corresponding to the response on a sheet previously prepared, and started the next trial.

265

266

**Figure 2 here**

267 
After the end of the auditory task, only Italian participants were required to fill the 58 -item

269 Italian version of Profile Of Mood State (POMS). In this questionnaire, a global score, called

270 Total Mood Disturbance (TMD), is calculated from the sub-scores as follows: Tension +

271 Depression + Anger + Fatigue + Confusion - Vigour +100 . This type of testing had already been

272 used in monitoring the mood state in sports and hypoxic environments (Peri, Scarlata \&

273 Barbarito, 2000; Kenttä, Hassmén \& Raglin, 2006; Karinen \& Tuomisto, 2017).

274

\section{Statistical analyses}

Statistical analyses were carried out using the R-based open-source software Jamovi (The

277

278

279

Jamovi project. jamovi (Version 1.0) [Computer Software]. https://www.jamovi.org 2019) for General Linear Model (GLM) Mediation Analyses, and by means of Statistica 8.0.550 (StatSoft. Inc., Tulsa, OK, USA) for Analyses of Variance (ANOVAs).

Mean pleasantness evaluations for consonant and dissonant chords expressed at the lower altitude (Kathmandu) and at the higher altitude (Ghunsa and Lhonak) were considered separately. Moreover, the difference of pleasantness evaluation between consonant and dissonant chords was also calculated, obtaining an overall measure in which negative scores corresponded to dissonance preference, positive scores corresponded to consonance preference and a score of 0 corresponded to an equal preference between consonant and dissonant chords.

A first GLM Mediation Analysis was used to test the mediation role of age and years of schooling, with Consonance-Dissonance difference, Consonance pleasantness, and Dissonance pleasantness as the dependent variables and Ethnicity (Italian, Nepalese, Sherpa), Listening to Western music (yes vs no) or Playing music (yes vs no) as the independent variables. A second GLM Mediation Analysis was used on the Italian sample to test TMD (from POMS 
291 questionnaire) as a mediator, with Consonance pleasantness, Dissonance pleasantness, and

292 Consonance-Dissonance difference as the dependent variables and Altitude (low, high) as the

293 independent variable. Both mediations were carried out with the jAMM (Advanced Mediation

294 Models) suite for Jamovi software (https://jamovi-amm.github.io/index.html).

295 Then, in a mixed-model ANOVA, Ethnicity (Italian, Nepalese, Lowlander-Sherpa) was

296 considered as a between-subjects factor, and Chord (consonant, dissonant) was used as a within-

297 subject factor. Pleasantness evaluations recorded at the lower altitude were used as the dependent

298 variable. In a second ANOVA, Ethnicity (Italian, Nepalese, Highlander-Sherpa) was considered

299 as a between-subjects factor, Chord (consonant, dissonant) was used as a within-subject factor,

300 and pleasantness evaluations recorded at the higher altitude were used as the dependent variable.

301 This separation was necessary because Italian and Nepalese participants carried out the task at

302 both altitudes, whereas Sherpa participants who carried out the task at lower and higher altitudes

303 were two different subgroups. For this reason, in a third ANOVA, only Italian and Nepalese

304 participants were included, and both Chord (consonant, dissonant) and Altitude (low, high) were

305 used as within-subject factors, whereas Ethnicity (Italian, Nepalese) was used as between-subject

306 factor. Pleasantness evaluations were used as the dependent variable. Finally, considering only

307 the Sherpa subgroups, a fourth ANOVA was carried out, by using the pleasantness evaluation as

308 the dependent variable, and by using Chord (consonant, dissonant) as within-subject factor and

309 Altitude (low, high) as between-subject factor. In all ANOVAs post-hoc tests were carried out by

310 means of Duncan test.

311

312

Results

313

The first GLM Mediation Analysis revealed that, among the variables of Ethnicity, Playing 
314 music and Listening to Western music, with Age and Schooling as mediators, the only one that 315 significantly predicted Consonance-Dissonance difference was Listening to Western music $(\beta$

316 weight $=0.572, p<0.001)$, mainly and significantly with a direct effect $(\beta=0.441, p=0.021)$, not

317 with the mediation of Age $(\beta=0.010, p=0.813)$ nor Schooling $(\beta=0.121, p=0.241)$. Listening

318 to Western music also predicted Consonance pleasantness $(\beta=0.706, p=0.009)$ without the

319 mediation of Age or schooling, whereas did not predict Dissonance pleasantness $(\beta=0.463, p=$ 320 0.219).

321 The second GLM Mediation Analysis, carried out to test the role of TMD (from POMS 322 questionnaire), revealed that altitude significantly predicted TMD $(\beta=-0.550, \mathrm{p}=0.023)$, with

323 lower TMD values at high $(93.50 \pm 9.16)$ with respect to low altitude $(115.83 \pm 24.65)$. No

324 significant prediction was found for TMD with respect to any of the predictor variables

325 (Consonance, Dissonance, Difference between consonance and dissonance), nor was any

326 mediation revealed (see Figure 3); as aforementioned, the TMD test was only done for the Italian 327 group.

**Figure 3 here**

330

In the first ANOVA (lower altitude), the main effect of Chord was significant $\left(F_{(1,14)}=\right.$ 332 40.62, $\left.p<0.001, \eta_{p}{ }^{2}=0.74\right)$, confirming the "consonance effect", with higher pleasantness scores for consonant chords $(\mathrm{M} \pm \mathrm{SEM}: 2.63 \pm 0.15)$ with respect to dissonant chords $(2.1 \pm$ $\left.<0.001, \eta_{p}{ }^{2}=0.64\right)$ and post-hoc tests confirmed the consonance effect in both Italian and

336 Sherpa samples ( $p<0.001$ for both comparisons), but not in Nepalese participants $(p=0.82)$. 

significance $\left(F_{(1,14)}=3.53, p=0.081\right)$. The main effect of Ethnicity was significant $\left(F_{(2,14)}=\right.$ 3.82, $\left.p=0.048, \eta_{p}{ }^{2}=0.35\right)$, revealing lower pleasantness scores for Sherpa $(1.85 \pm 0.25)$ with respect to both Italian $(2.45 \pm 0.25, p=0.028)$ and Nepalese $(2.46 \pm 0.08, p=0.033)$ participants. Finally, the interaction was also significant $\left(F_{(2,14)}=10.88, p=0.001, \eta_{p}^{2}=0.61\right)$, and post-hoc comparison showed that only in the Italian sample consonant chords $(2.94 \pm 0.31)$ were judged as more pleasant than dissonant chords $(1.97 \pm 0.29, p<0.001)$. Furthermore, consonant chords received lower pleasantness judgments by Sherpa $(1.74 \pm 0.17)$ compared to both Italian $(2.94 \pm 0.31, p<0.001)$ and Nepalese $(2.42 \pm 0.12, p=0.038)$ participants. and Nepalese groups, further confirmed the consonance effect, as shown by the main effect of Chord $\left(F_{(1,20)}=24.64, p<0.001, \eta_{p}{ }^{2}=0.55\right)$, with higher pleasantness scores for consonant $(2.61$ $\pm 0.12)$ than for dissonant $(2.17 \pm 0.11)$ chords. Importantly, the interaction between Ethnicity and Chord was significant $\left(F_{(1,20)}=32.33, p<0.001, \eta_{p}{ }^{2}=0.62\right.$; Figure 4$)$, and post-hoc comparisons showed that the consonant effect was due exclusively to the Italian group ( $p<$ 0.001), who rated consonant chords as more pleasant than dissonant chords. Accordingly, consonant chords were evaluated as more pleasant by Italian than Nepalese participants $(p=$ 0.03), whereas dissonant chords were evaluated as less pleasant by Italian than Nepalese participants $(p=0.025)$. The other main effects and interactions were not significant, revealing no effect of the altitude on the auditory test. 
either at lower or higher altitude, showed a trend toward the consonant effect which failed to reach statistical significance $\left(F_{(1,8)}=3.23, p=0.056\right)$. Only the interaction between Altitude and Chord was significant $\left(F_{(1,20)}=17.22, p=0.003, \eta_{p}{ }^{2}=0.68\right.$; Figure 5), revealing a consonance

effect only in the lowlander group $(p=0.002)$, but not in the highlander group $(p=0.21)$.

Furthermore, consonant chords were judged as more pleasant by lowlanders than by highlanders $(p=0.018)$

\section{Discussion}

The first important finding of the present cross-cultural pilot study supports the cultural origin of music pleasantness: the consonance effect was confirmed in the Italian sample and it was not found in the Nepalese sample. This first result would exclude the possibility that preference for consonant music is innate and biologically determined, because if this hypothesis were true we should have found a similar preference in all the samples tested. We were able to test this hypothesis because in Nepalese and Sherpa groups there were participants who declared not to listen to Western music. While it can be assumed for Highlander Sherpas, living in remote regions, one may be wondering why there were diverse habits between lowlander Sherpas and Nepalese. We think the reason of the difference to be related to the working habit: if Sherpas living in Kathmandu were used to be involved directly in commercial or mountaineering activities with foreign individuals, Nepalese porters had a secondary role in the link with mountaineers or trekkers. 
384 showed that a consonance effect was present in lowlander Sherpas, namely the subsample who

385 declared to listen to Western music, but the effect was completely absent in highlander Sherpas,

386 the subsample who declared to listen to music less frequently and, importantly, not to listen to

387 Western music. This latter result is the most important of the present study, because it shows that

388 music exposure is the key factor determining the consonance effect: even if they belong to the

389 same ethnicity, only Sherpas often exposed to Western music showed a consonance effect, even

390 if to a lesser degree than the Italian sample. This conclusion is statistically confirmed also by the

391 mediation analysis, revealing that listening to Western music influenced the consonance effect,

392 independently from the ethnical group. Indeed, listening to Western music predicted consonance-

393 dissonance difference, as well as consonance pleasantness, and did not predict dissonance

394 pleasantness. In addition, neither age nor schooling mediated these results, allowing us to affirm

395 that the only effect which influenced the consonance effect was the exposure to Western music.

396 In the present study we also explored the possible effect of some demographic features on

397 the consonance effect, such as age, schooling and playing music: the results revealed that none of

398 these variables influences music pleasantness, stressing that the exposure to a particular kind of

399 music is likely the most salient variable responsible for the consonance effect. It has to be

400 underlined that previous evidence revealed that preference for consonance is related to musical

401 experience, showing a higher preference for harmonic chords in participants who had music

402 training, even if aesthetic judgments for dissonant chords were not influenced by musical

403 training (McDermott, Lehr \& Oxenham, 2010). Moreover, in a cross-cultural study with Western

404 and African (Cameroon) participants, Fritz and colleagues (Fritz et al., 2009) found no group

405 differences in judging both emotional content (happy, sad, fearful) and pleasantness of Western 
406

407 Japanese listeners were found by Butler and Daston (1968), whereas a preference for consonance

and African music. Similarly, no difference in chords preference between American and vs dissonance was described in newborns (Trainor, Tsang \& Cheung, 2002) as well as in non-

409 human species (Fishman et al., 2001; Chiandetti \& Vallortigara, 2011), thus suggesting a biological basis of music processing, independent from the culture.

Even if we did not have specific hypotheses in this regard, we also considered altitude hypoxia as a possible influencing variable, because of the well-known affecting role of such condition in sensory systems and cognitive performance. Indeed, among the numerous evidence on the effects of hypoxia, nasal, ear, and throat complaints (Cingi, Erkan \& Rettinger, 2010), short-term and long-term eye diseases (Jha, 2012), increases of the olfactory threshold (Ruffini et al., 2015), and impairment of attentional performances (Limmer \& Platen, 2018) have been shown. Instead, our results showed that music pleasantness was not influenced by altitude. Other mood dimensions were affected by hypoxia, as resembled by the POMS: the Italian sample revealed lower mood disturbance scores (index of tension, depression, anger, fatigue and confusion) at higher compared to lower altitudes. We speculate that this difference could be due to the fact that once climbed the mountain, the more positive mood can be due to a kind of gratification compared to the possible concern before the climb. This result agrees with the findings of Karinen and Tuomisto, who reported that during a prolonged expedition in Everest mountain well-motivated participants were capable to maintain a good mood state (Karinen \& Tuomisto, 2017); on the contrary, Stavrou and colleagues, who reported that hypoxia was capable to negatively affect the mood, exacerbating the effect of bed rest and ambulatory confinement (Stavrou et al., 2018). Other evidence linked mood and altitude hypoxia, affirming that mood states were adversely affected by duration and level of altitude, but with a main role of 
429 physical exertion (Shukitt-Hale \& Lieberman, 1996). Physical exercise is known to affect mood

430 state (Di Corrado et al., 2014). In this regard, Murgia and colleagues recently found that physical

431 exertion alone may negatively affect mood states, mainly in low-performing athletes (Murgia et

432 al., 2016). Compared to these latest results, participants of the present study were not particularly

433 affected by physical exertion, nor they experienced an unsuccessful performance. Several

434 physiological and psychological variables can support the lower mood disturbance found at high

435 altitude in the current study. According to the self-efficacy hypothesis, gratification might have

436 played a role: the degree of reward, combined with an athletic success such as trekking to a

437 Himalayan base camp, would positively have affected the mood state (Eldar et al., 2018). Among

438 the biochemical factors, endorphins might be evoked - considering the positive association with

439 long term high altitude exposure (Appenzeller \& Wood, 1992) - as well as modulations of

440 hypothalamic-pituitary-adrenal axis, mTOR signaling, and serotonin release (Mikkelsen et al.,

441 2017). Moreover, Heinrich and colleagues recently affirmed that acclimatization plays a big role

442 in cognitive and mood alterations during a sojourn at altitude (Heinrich et al., 2019). To be noted

443 that none of our participants suffered remarkable altitude sickness symptoms. In summary,

444 neither strenuous physical exertion, nor ineffective acclimatization, nor forced confinement

445 stressed our participants. Thus, from these findings and from our results, we support the idea that

446 hypoxia may increase the mood disturbance during non-rewarding conditions or in association

447 with altitude sickness symptoms or strenuous physical exercise, but that hypoxia per se, or

448 hypoxia during motivating conditions does not promote mood disturbances.

449 The present results, suggesting a crucial role of music exposure on music pleasantness, are

450 largely in accordance with those described by McDermott and colleagues (McDermott et al.,

451 2016) who tested US, Bolivian and Amazonian participants. In their study, the authors found that 
452 Western participants judged consonant chords as more pleasant than dissonant chords, and they

453 found that Amazonians - not exposed to Western culture - did not show this preference, and

454 Bolivians' scores were halfway. This pattern of results is confirmed also by the present study, in

455 which the preference for consonant over dissonant chords was high for Italians, it was absent for

456 Nepalese and Sherpa not exposed to Western culture, but it was present (in a lesser degree

457 compared to Italians) in Sherpa often exposed to Western music. Compared to their findings, the

458 present study adds the evidence of null effects of other demographic information on music

459 preference, as well as it shows a null effect of hypobaric hypoxia on the pleasantness of music.

460 We should highlight that, due to the low sample size tested in this pilot study, which also

461 led to an incomplete design (for instance, not all of the three groups were tested both at lower

462 and higher altitude), and to the absence of control tasks, caution is needed in the generalization

463 of the present results. Nevertheless, we should also highlight that our findings strictly resemble

464 those recently described by McDermott and colleagues (2016). It has to be noted, moreover, that

465 the relatively low sample size is also due to the difficulty in testing participants belonging to

466 remote cultures, and mainly to the fact that the test was administered at different altitudes,

467 including Lhonak (4780 m); the same contextual factors limited the possibility to perform

468 control tasks and to test a full factorial design (i.e., each group of Sherpas was tested where

469 participants lived, and we cannot obtain data of lowlanders/highlanders at high/low altitude,

470 respectively). However, these limitations are typical of field studies in extreme environments. In

471 this frame, we underlie the need of more cross-cultural evidence in order to assess the possible

472 origin of music pleasantness. Starting from the results collected in this pilot study, we can

473 exclude the effect of hypoxia on pleasantness evaluation, and this result will allow to avoid one

474 of the aspect which made it difficult to enlarge the sample, namely altitude, allowing to focus on 
475 music habits (i.e., listening to Western music) more than on living altitude or testing altitude.

476 Similarly, the fact that the mood seems to be related only to altitude (hypoxia) allows us to

477 conclude that music pleasantness is not influenced by this variable, but also in this case caution

478 is needed because, due to a technical issue, POMS was administered only to the Italian sample.

479 Despite we did not carry out a control task, our Nepalese sample demonstrated to have

480 understood the instructions, from a brief familiarization with the experimental setting and

481 feedback from the native speakers who supported us translating the instructions.

482 To summarize, we could consider these pilot results as a further evidence in favor of the

483 crucial role of music exposure in music preference, having found that neither physiological

484 changes possibly due to altitude, nor demographic differences influence this preference. Cross-

485 cultural studies represent unique opportunities to test the biological bases of the most disparate

486 human behaviors, and the present results add further evidence supporting that music pleasantness

487 is not universally shared, but strongly depends upon habits.

488

489 
490 References

491 Appenzeller O, Wood SC. 1992. Peptides and exercise at high and low altitudes. International Journal of

492 Sports Medicine 13 Suppl 1:S135-140. DOI: 10.1055/s-2007-1024618.

493 Bowling DL, Hoeschele M, Gill KZ, Fitch WT. 2017. The Nature and Nurture of Musical Consonance.

494 Music Perception: An Interdisciplinary Journal 35:118-121. DOI: 10.1525/mp.2017.35.1.118.

495 Bowling DL, Purves D. 2015. A biological rationale for musical consonance. Proceedings of the National

496 Academy of Sciences of the United States of America 112:11155-11160. DOI: 10.1073/pnas.1505768112.

497 Butler JW, Daston PG. 1968. Musical consonance as musical preference: A cross-cultural study. Journal

498 of General Psychology 79:129-142. DOI: 10.1080/00221309.1968.9710460.

499 Castellano MA, Bharucha JJ, Krumhansl CL. 1984. Tonal hierarchies in the music of North India.

500 Journal of Experimental Psychology: General 113:394-412. DOI: 10.1037/0096-3445.113.3.394.

501 Cazden N. 1980. The Definition of Consonance and Dissonance. International Review of the Aesthetics

502 and Sociology of Music 11:123-168. DOI: 10.2307/836494.

503 Chiandetti C, Vallortigara G. 2011. Chicks like consonant music. Psychological Science 22:1270-1273.

504 DOI: 10.1177/0956797611418244.

505 Cingi C, Erkan AN, Rettinger G. 2010. Ear, nose, and throat effects of high altitude. European archives

506 of oto-rhino-laryngology: official journal of the European Federation of Oto-Rhino-Laryngological

507 Societies (EUFOS): affiliated with the German Society for Oto-Rhino-Laryngology - Head and Neck

508 Surgery 267:467-471. DOI: 10.1007/s00405-009-1016-6.

509 Cross I. 2003. Music as a biocultural phenomenon. Annals of the New York Academy of Sciences

510 999:106-111. DOI: 10.1196/annals.1284.010.

511 Di Corrado D, Agostini T, Bonifazi M, Perciavalle V. 2014. Changes in mood states and salivary cortisol

512 levels following two months of training in elite female water polo players. Molecular Medicine Reports

513 9:2441-2446. DOI: 10.3892/mmr.2014.2115.

514 Dinnerstein N, Alter A. 2018. Introduction: Popular Music Across the Himalayas. HIMALAYA, the

515 Journal of the Association for Nepal and Himalayan Studies 38.

516 Eldar E, Roth C, Dayan P, Dolan RJ. 2018. Decodability of Reward Learning Signals Predicts Mood

517 Fluctuations. Current Biology 28:1433-1439.e7. DOI: 10.1016/j.cub.2018.03.038.

518 Fishman YI, Volkov IO, Noh MD, Garell PC, Bakken H, Arezzo JC, Howard MA, Steinschneider M.

519 2001. Consonance and dissonance of musical chords: neural correlates in auditory cortex of monkeys and

520 humans. Journal of Neurophysiology 86:2761-2788. DOI: 10.1152/jn.2001.86.6.2761.

521 Fritz T, Jentschke S, Gosselin N, Sammler D, Peretz I, Turner R, Friederici AD, Koelsch S. 2009.

522 Universal recognition of three basic emotions in music. Current biology: $C B$ 19:573-576. DOI:

523 10.1016/j.cub.2009.02.058.

Peer] reviewing PDF | (2020:03:46935:2:0:NEW 17 May 2020) 
524 Golja P, Kacin A, Tipton MJ, Mekjavic IB. 2005. Moderate hypoxia does not affect the zone of thermal

525 comfort in humans. European Journal of Applied Physiology 93:708-713. DOI: 10.1007/s00421-004-

526 1306-z.

527 Greene PD. 2003. Nepal's Lok Pop music: Representations of the folk, tropes of memory, and studio

528 technologies. Asian Music 34:43-65.

529 Harrison PMC, Pearce MT. 2020. Simultaneous Consonance in Music Perception and Composition.

530 Psychological Review 127:216-244. DOI: 10.1037/rev0000169.

531 Heinrich EC, Djokic MA, Gilbertson D, DeYoung PN, Bosompra N-O, Wu L, Anza-Ramirez C, Orr JE, 532 Powell FL, Malhotra A, Simonson TS. 2019. Cognitive function and mood at high altitude following 533 acclimatization and use of supplemental oxygen and adaptive servoventilation sleep treatments. PLOS 534 ONE 14:e217089. DOI: 10.1371/journal.pone.0217089.

535 Henderson D. 2002. "Who Needs 'The Folk'?” A Nepali Remodeling Project. Asian Music 34:19-42.

536 Higgins KM. 2012. The Music between Us. University of Chicago Press.

537 Huppertz T, Freiherr J, Olzowy B, Kisser U, Stephan J, Fesl G, Haegler K, Feddersen B, Fischer R, Mees 538 K, Becker S. 2018. Reduction of olfactory sensitivity during normobaric hypoxia. Auris, Nasus, Larynx 539 45:747-752. DOI: 10.1016/j.anl.2017.11.001.

540 Jha KN. 2012. High Altitude and the Eye. Asia-Pacific Journal of Ophthalmology (Philadelphia, Pa.)

541 1:166-169. DOI: 10.1097/APO.0b013e318253004e.

542 Karinen HM, Tuomisto MT. 2017. Performance, Mood, and Anxiety During a Climb of Mount Everest.

543 High Altitude Medicine \& Biology 18:400-410. DOI: 10.1089/ham.2017.0033.

544 Kenttä G, Hassmén P, Raglin JS. 2006. Mood state monitoring of training and recovery in elite kayakers. 545 European Journal of Sport Science 6:245-253. DOI: 10.1080/17461390601012652.

546 Koelsch S, Boehlig A, Hohenadel M, Nitsche I, Bauer K, Sack U. 2016. The impact of acute stress on 547 hormones and cytokines and how their recovery is affected by music-evoked positive mood. Scientific

548 Reports 6:1-11. DOI: 10.1038/srep23008.

549 Limmer M, Platen P. 2018. The influence of hypoxia and prolonged exercise on attentional performance 550 at high and extreme altitudes: A pilot study. PLOS ONE 13:e205285. DOI:

551 10.1371/journal.pone.0205285.

552 Maher TF. 1976. "Need for Resolution" Ratings for Harmonic Musical Intervals: A Comparison between 553 Indians and Canadians. Journal of Cross-Cultural Psychology 7:259-276. DOI:

$55410.1177 / 002202217673001$.

555 Malm W. 1996. Music Cultures of the Pacific, the Near East, and Asia, 3rd Edition. Pearson.

556 McDermott J, Hauser M. 2004. Are consonant intervals music to their ears? Spontaneous acoustic 557 preferences in a nonhuman primate. Cognition 94:B11-21. DOI: 10.1016/j.cognition.2004.04.004. 
558 McDermott JH, Lehr AJ, Oxenham AJ. 2010. Individual Differences Reveal the Basis of Consonance.

559 Current biology: CB 20:1035-1041. DOI: 10.1016/j.cub.2010.04.019.

560 McDermott JH, Schultz AF, Undurraga EA, Godoy RA. 2016. Indifference to dissonance in native

561 Amazonians reveals cultural variation in music perception. Nature 535:547-550. DOI:

$56210.1038 /$ nature18635.

563 Messner GF. 1981. The Two-Part Vocal Style on Baluan Island Manus Province, Papua New Guinea.

564 Ethnomusicology 25:433-446. DOI: 10.2307/851553.

565 Mikkelsen K, Stojanovska L, Polenakovic M, Bosevski M, Apostolopoulos V. 2017. Exercise and mental 566 health. Maturitas 106:48-56. DOI: 10.1016/j.maturitas.2017.09.003.

567 Moore LG. 2001. Human Genetic Adaptation to High Altitude. High Altitude Medicine \& Biology 2:257568 279. DOI: $10.1089 / 152702901750265341$.

569 Murgia M, Forzini F, Filho E, DI Fronso S, Sors F, Bertollo M, Agostini T. 2016. How do mood states 570 change in a multi-stage cycling competition? Comparing high and low performers. The Journal of Sports 571 Medicine and Physical Fitness 56:336-342.

572 Peri A, Scarlata C, Barbarito M. 2000. Preliminary Studies on the Psychological Adjustment in the Italian 573 Antarctic Summer Campaigns. Environment and Behavior 32:72-83. DOI:

$57410.1177 / 00139160021972432$.

575 Plantinga J, Trehub SE. 2014. Revisiting the innate preference for consonance. Journal of Experimental 576 Psychology. Human Perception and Performance 40:40-49. DOI: 10.1037/a0033471.

577 Popescu T, Neuser MP, Neuwirth M, Bravo F, Mende W, Boneh O, Moss FC, Rohrmeier M. 2019. The 578 pleasantness of sensory dissonance is mediated by musical style and expertise. Scientific Reports 9:1-11. 579 DOI: $10.1038 / \mathrm{s} 41598-018-35873-8$.

580 Prajapati SR. 2018. Traditional, Folk, Fusion, and conFusion: Music and Change in the Newar 581 Communities of Nepal. Thesis Thesis.

582 Prete G, D’Anselmo A, Brancucci A, Tommasi L. 2019. Unilateral hf-tRNS over the temporal cortex 583 does not influence pleasantness of musical chords. Applied Acoustics 154:135-137. DOI:

584 10.1016/j.apacoust.2019.04.035.

585 Prete G, Fabri M, Foschi N, Brancucci A, Tommasi L. 2015. The "consonance effect" and the 586 hemispheres: A study on a split-brain patient. Laterality 20:257-269. DOI:

587 10.1080/1357650X.2014.959525.

588 Ruffini R, Di Giulio C, Verratti V, Pokorski M, Fanò-Illic G, Mazzatenta A. 2015. Adaptation of 589 olfactory threshold at high altitude. Advances in Experimental Medicine and Biology 837:19-22. DOI: 590 10.1007/5584_2014_70.

591 Segall MH, Dasen PR, Berry JW, Poortinga YH. 1990. Human behavior in global perspective: An 592 introduction to cross-cultural psychology. Elmsford, NY, US: Pergamon Press. 
593 Shukitt-Hale B, Lieberman HR. 1996. The Effect of Altitude on Cognitive Performance and Mood States. 594 In: Nutritional Needs In Cold And In High-Altitude Environments: Applications for Military Personnel in 595 Field Operations. National Academies Press (US),

596 Stavrou NAM, Debevec T, Eiken O, Mekjavic IB. 2018. Hypoxia Exacerbates Negative Emotional State 597 during Inactivity: The Effect of 21 Days Hypoxic Bed Rest and Confinement. Frontiers in Physiology 598 9:26. DOI: 10.3389/fphys.2018.00026.

599 Terhardt E. 1984. The concept of musical consonance: A link between music and psychoacoustics. Music 600 Perception 1:276-295. DOI: 10.2307/40285261.

601 Trainor LJ, Tsang CD, Cheung VHW. 2002. Preference for Sensory Consonance in 2- and 4-Month-Old 602 Infants. Music Perception: An Interdisciplinary Journal 20:187-194. DOI: 10.1525/mp.2002.20.2.187.

603 Tramo MJ, Cariani PA, Delgutte B, Braida LD. 2001. Neurobiological foundations for the theory of 604 harmony in western tonal music. Annals of the New York Academy of Sciences 930:92-116. DOI: 605 10.1111/j.1749-6632.2001.tb05727.x.

606 Tsukamoto A. 1983. The Music of Tibetan Buddhism in Ladakh: The Musical Structure of Tibetan 607 Buddhist Chant in the Ritual Bskan-gso of the Dge-Lugs-pa Sect. Yearbook for Traditional Music 608 15:126-140. DOI: 10.2307/768646.

609 West JB. 2016. Early history of high-altitude physiology. Annals of the New York Academy of Sciences 610 1365:33-42. DOI: 10.1111/nyas.12719. 


\section{Acknowledgments}

613 Authors thank Ghunsa Hospital (Ghunsa, Nepal) for the assistance in logistics, all the Italian 614 trekkers, Nepalese porters and Sherpas. This work was supported by a grant of Department of 615 Psychological, Health and Territorial Sciences, “G. d'Annunzio” University of Chieti-Pescara, 616 Italy, to Vittore Verratti.

617

618 Authors contribution

619 Conceptualization, G.P., D.B., V.V. and L.T.; Methodology, G.P., D.B., V.V., A.A. and L.T.; 620 Investigation, D.B., V.V. and P.R.; Formal Analyses, G.P. and D.B.; Writing - Original Draft, 621 G.P., D.B., V.V. and L.T.; Writing - Review \& Editing, G.P., D.B., V.V., A.A., P.R. and L.T.; 622 Funding Acquisition, V.V. and L.T.; Project administration, V.V.; Resources, G.P., D.B., V.V., 623 A.A., P.R. and L.T.; Supervision, V.V. and L.T.

625 Competing interests

626 Luca Tommasi is an Academic Editor for PeerJ. 
629 Table 1: Self-reported demographic data: Eth (Ethnicity: ITA = Italian, NEP = Nepalese, SHEh = 630 highlander Sherpas; SHEl = lowlander Sherpa); Liv (Living altitude: L = Low altitude, H = High 631 altitude); Test (Testing altitude: $\mathrm{L}=$ Low altitude, $\mathrm{H}=$ High altitude); Sex $(\mathrm{F}=$ female, $\mathrm{M}=$ male); Age 632 (years); Sch (schooling: years of instructions); Mus (YES = musician, NO = not musician); Y/Mus (years 633 of music study); West (YES = listening to Western music; NO = not listening to Western music); Freq

634 (frequency of Western music listening); Lang (number of foreign language spoken); Eng (Knowledge of 635 English language: $\mathrm{YES}=$ English speaker, $\mathrm{NO}=$ non-English speaker); Hand (handedness: $\mathrm{L}=$ left, $\mathrm{R}=$ 636 right).

637

638 


\section{Figure captions}

640 Figure 1: Altimetric plan of "Kanchenjunga Exploration \& Physiology" project. The continuous 641 line represents the effective trek. The plain data points represent the nights spent in the related 642 location. The empty circular data point at Kanchenjunga North Base Camp (KJ N-Base Camp) 643 represents the highest altitude reached by participants throughout the trek. The triangular data 644 points represent the measurements.

645

646 Figure 2: Actual experimental setting at high altitude.

647

648 Figure 3: Mediation analysis of Total Mood Disturbance (TMD) from Profile Of Mood State 649 (POMS) questionnaire, on the relation between altitude and music pleasantness. Values 650 displayed are $\beta$ weights. Dotted lines represent the indirect effects (i.e. those mediated by TMD), 651 while the whole lines represent the direct effects. Panel A shows the analysis on Consonance 652 (Cons.), panel B on Dissonance (Diss.) and panel C on the difference Consonance - Dissonance 653 (Diff.).

654

Figure 4: Pane A) Interaction between Ethnicity (Italian, Nepalese) and Chord (consonant, 656 dissonant), on the pleasantness judgments; asterisks show significant comparisons $(p<0.05)$;

657 Panel B) pleasantness judgments for consonant and dissonant chords expressed by each 658 participant of the two groups; Panel C) pleasantness judgments by Italian and Nepalese 659 participants expressed for each chord. Judgments were expressed on a 1-4 Likert scale. Bars 660 represent standard errors. 
662 Figure 5: Panel A) Interaction between Altitude (low altitude: lowlander subsample, high

663 altitude: highlander subsample) and Chord (consonant, dissonant), on the pleasantness

664 judgments; asterisks show significant comparisons $(p<0.05)$. Panel B) pleasantness judgments

665 for consonant and dissonant chords expressed by each participant of the two subgroups; Panel C)

666 pleasantness judgments by lowlander and highlander Sherpas expressed for each chord.

667 Judgments were expressed on a 1-4 Likert scale. Bars represent standard errors.

668

669 Supplementary materials

670 Twelve consonant and twelve dissonant chords used in the task as stimuli. 


\section{Table $\mathbf{1}$ (on next page)}

Self-reported demographic data

Self-reported demographic data: Eth (Ethnicity: ITA = Italian, NEP = Nepalese, SHEh = highlander Sherpas; SHEI = lowlander Sherpa); Liv (Living altitude: $L=$ Low altitude, $H=$ High altitude); Test (Testing altitude: $L=$ Low altitude, $H=$ High altitude); Sex $(F=$ female, $M$ $=$ male); Age (years); Sch (schooling: years of instructions); Mus (YES = musician, NO = not musician); Y/Mus (years of music study); West (YES = listening to Western music; NO = not listening to Western music); Freq (frequency of Western music listening); Lang (number of foreign language spoken); Eng (Knowledge of English language: YES = English speaker, NO = non-English speaker); Hand (handedness: $L=$ left, $R=$ right). 


\section{Table 1}

\begin{tabular}{|c|c|c|c|c|c|c|c|c|c|c|c|c|c|}
\hline $\mathbf{N}$ & Eth & Liv & Test & Sex & Age & Sch & Mus & Y/Mus & West & Freq & Lang & Eng & Hand \\
\hline 1 & ITA & \multirow{6}{*}{$\mathrm{L}$} & \multirow{6}{*}{ L-H } & $\mathrm{F}$ & 36 & 21 & YES & 30 & YES & often & 2 & YES & $\mathrm{R}$ \\
\hline 2 & ITA & & & $\mathrm{M}$ & 63 & 18 & NO & 0 & YES & always & 0 & $\mathrm{NO}$ & $\mathrm{R}$ \\
\hline 3 & ITA & & & $\mathrm{M}$ & 59 & 13 & $\mathrm{NO}$ & 0 & YES & always & 1 & $\mathrm{NO}$ & $\mathrm{R}$ \\
\hline 4 & ITA & & & $\mathrm{M}$ & 25 & 17 & $\mathrm{NO}$ & 0 & YES & always & 1 & YES & $\mathrm{R}$ \\
\hline 5 & ITA & & & $\mathrm{M}$ & 32 & 20 & YES & 10 & YES & often & 2 & YES & $\mathrm{R}$ \\
\hline 6 & ITA & & & $\mathrm{M}$ & 48 & 22 & $\mathrm{NO}$ & 0 & YES & always & 2 & YES & $\mathrm{R}$ \\
\hline 7 & NEP & \multirow{6}{*}{$\mathrm{L}$} & \multirow{6}{*}{ L-H } & M & 26 & 12 & NO & 0 & YES & little & 3 & YES & $\mathrm{R}$ \\
\hline 8 & NEP & & & $\mathrm{M}$ & 18 & 10 & $\mathrm{NO}$ & 0 & $\mathrm{NO}$ & never & 0 & $\mathrm{NO}$ & $\mathrm{R}$ \\
\hline 9 & NEP & & & $\mathrm{M}$ & 39 & 0 & $\mathrm{NO}$ & 0 & $\mathrm{NO}$ & never & 0 & $\mathrm{NO}$ & $\mathrm{R}$ \\
\hline 10 & NEP & & & $\mathrm{M}$ & 40 & 7 & $\mathrm{NO}$ & 0 & $\mathrm{NO}$ & never & 2 & YES & $\mathrm{R}$ \\
\hline 11 & NEP & & & $\mathrm{M}$ & 30 & 4 & YES & 3 & $\mathrm{NO}$ & never & 2 & YES & $\mathrm{L}$ \\
\hline 12 & NEP & & & $\mathrm{M}$ & 29 & 0 & $\mathrm{NO}$ & 0 & $\mathrm{NO}$ & never & 1 & $\mathrm{NO}$ & $\mathrm{R}$ \\
\hline 13 & SHEh & \multirow{5}{*}{$\mathrm{H}$} & \multirow{5}{*}{$\mathrm{H}$} & $\mathrm{M}$ & 56 & 7 & NO & 0 & NO & never & 3 & YES & $\mathrm{R}$ \\
\hline 14 & SHEh & & & $\mathrm{M}$ & 23 & 12 & YES & 7 & YES & often & 3 & YES & $\mathrm{L}$ \\
\hline 15 & SHEh & & & $\mathrm{M}$ & 25 & 6 & $\mathrm{NO}$ & 0 & $\mathrm{NO}$ & never & 0 & $\mathrm{NO}$ & $\mathrm{R}$ \\
\hline 16 & SHEh & & & $\mathrm{M}$ & 54 & 0 & NO & 0 & $\mathrm{NO}$ & never & 1 & $\mathrm{NO}$ & $\mathrm{R}$ \\
\hline 17 & SHEh & & & $\mathrm{M}$ & 27 & 7 & YES & 6 & $\mathrm{NO}$ & never & 1 & $\mathrm{NO}$ & $\mathrm{R}$ \\
\hline 18 & SHEl & \multirow{5}{*}{$\mathrm{L}$} & \multirow{5}{*}{$\mathrm{L}$} & $\mathrm{M}$ & 37 & 16 & YES & $5-10$ & YES & often & 3 & YES & $\mathrm{R}$ \\
\hline 19 & SHEl & & & $\mathrm{M}$ & 23 & 12 & YES & $5-10$ & YES & often & 3 & YES & $\mathrm{R}$ \\
\hline 20 & SHEl & & & $\mathrm{M}$ & 29 & 6 & $\mathrm{NO}$ & 0 & YES & often & 4 & YES & $\mathrm{R}$ \\
\hline 21 & SHEl & & & $\mathrm{M}$ & 28 & 12 & $\mathrm{NO}$ & 0 & $\mathrm{NO}$ & never & 3 & YES & $\mathrm{R}$ \\
\hline 22 & SHEl & & & $\mathrm{M}$ & 26 & 10 & NO & 0 & YES & often & 3 & YES & $\mathrm{R}$ \\
\hline
\end{tabular}


Figure 1

Altimetric plan of "Kanchenjunga Exploration \& Physiology" project.

Altimetric plan of "Kanchenjunga Exploration \& Physiology" project. The continuous line represents the effective trek. The plain data points represent the nights spent in the related location. The empty circular data point at Kanchenjunga North Base Camp (KJ N-Base Camp) represents the highest altitude reached by participants throughout the trek. The triangular data points represent the measurements.

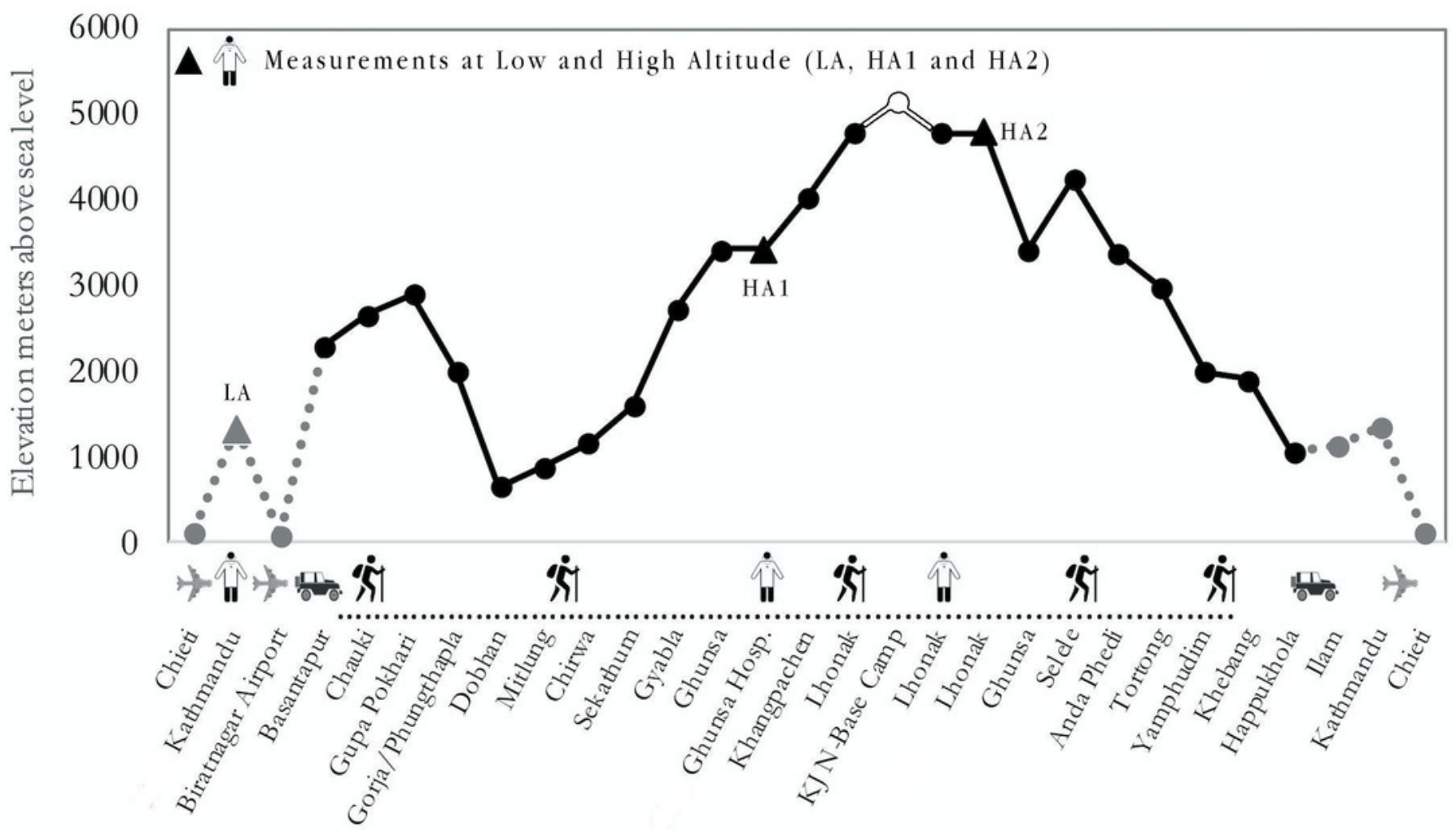


Figure 2

Actual experimental setting at high altitude.

Actual experimental setting at high altitude.

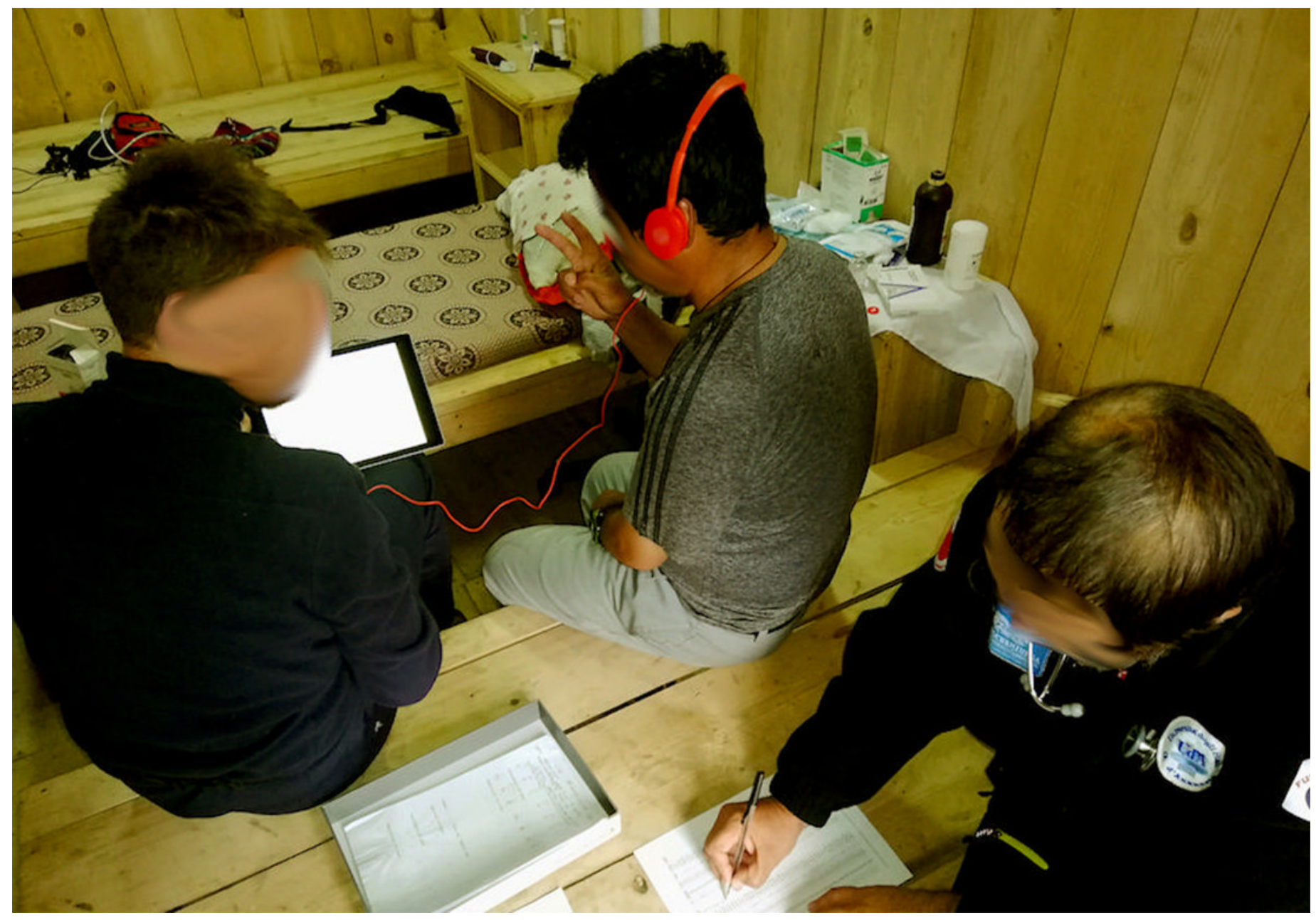




\section{Figure 3}

Mediation analysis of Total Mood Disturbance on the relation between altitude and music pleasantness.

Mediation analysis of Total Mood Disturbance (TMD) from Profile Of Mood State (POMS) questionnaire, on the relation between altitude and music pleasantness. Values displayed are $\beta$ weights. Dotted lines represent the indirect effects (i.e. those mediated by TMD), while the whole lines represent the direct effects. Panel A shows the analysis on Consonance (Cons.), panel B on Dissonance (Diss.) and panel C on the difference Consonance - Dissonance (Diff.).
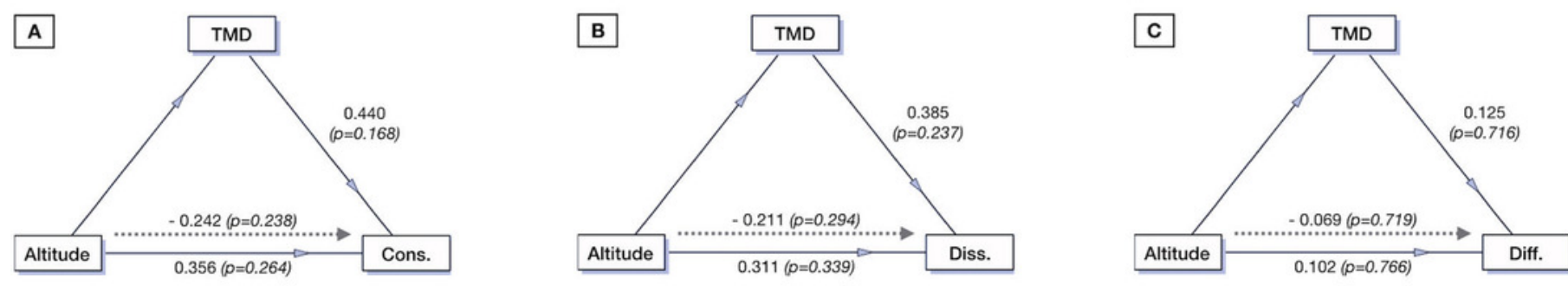


\section{Figure 4}

Interaction between Ethnicity and Chord.

Pane A) Interaction between Ethnicity (Italian, Nepalese) and Chord (consonant, dissonant), on the pleasantness judgments; asterisks show significant comparisons $(p<0.05)$; Panel B) pleasantness judgments for consonant and dissonant chords expressed by each participant of the two groups; Panel C) pleasantness judgments by Italian and Nepalese participants expressed for each chord. Judgments were expressed on a 1-4 Likert scale. Bars represent standard errors. 
A)

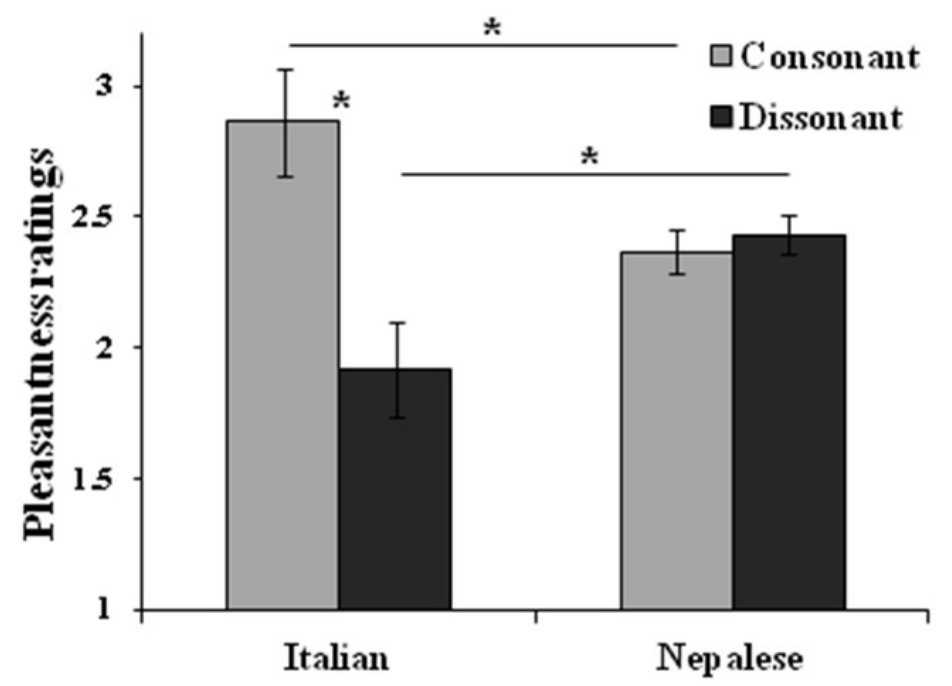

B)
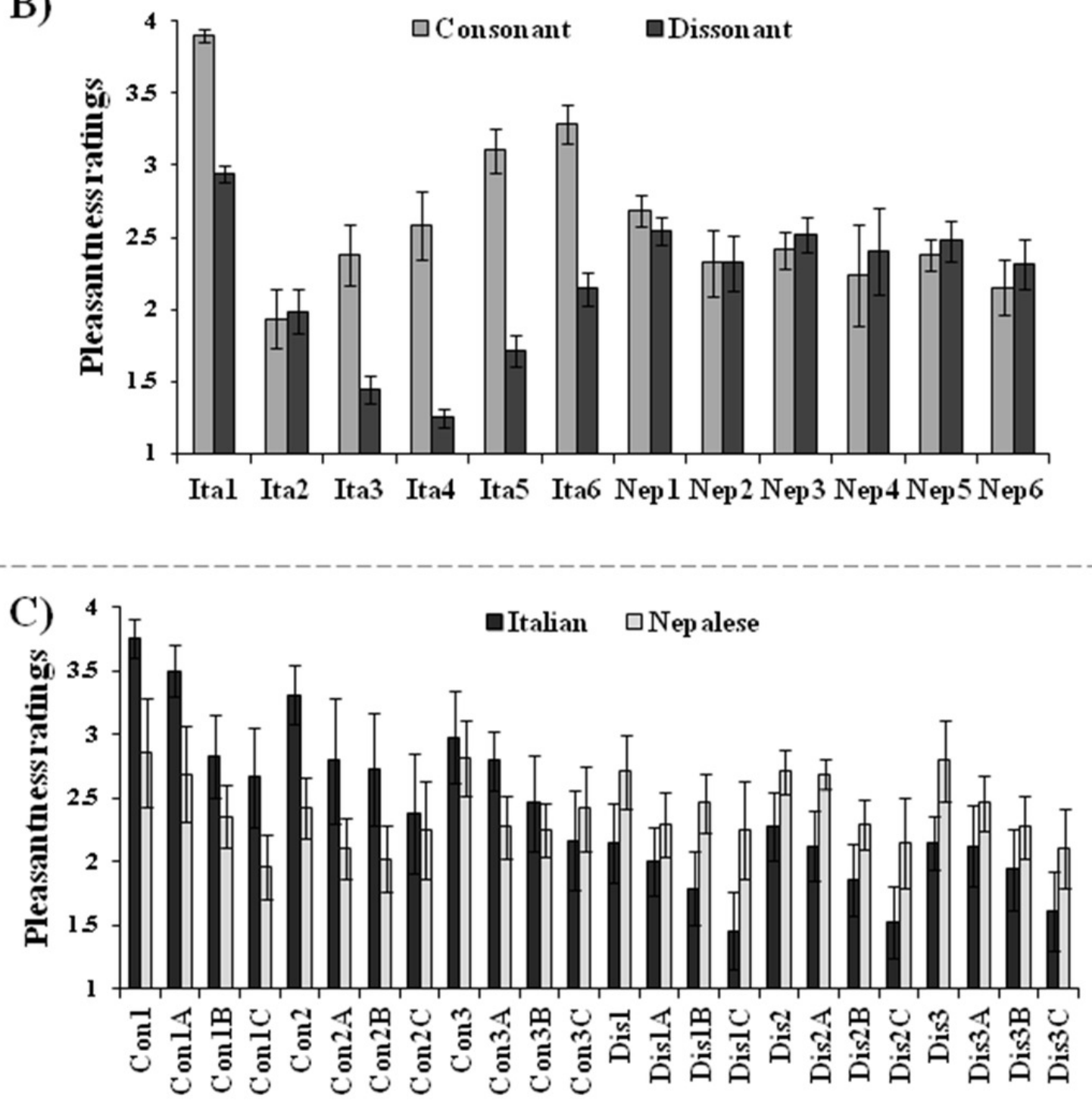


\section{Figure 5}

Interaction between Altitude and Chord.

Panel A) Interaction between Altitude (low altitude: lowlander subsample, high altitude:

highlander subsample) and Chord (consonant, dissonant), on the pleasantness judgments; asterisks show significant comparisons $(p<0.05)$. Panel B) pleasantness judgments for consonant and dissonant chords expressed by each participant of the two subgroups; Panel C) pleasantness judgments by lowlander and highlander Sherpas expressed for each chord. Judgments were expressed on a 1-4 Likert scale. Bars represent standard errors. 
A)

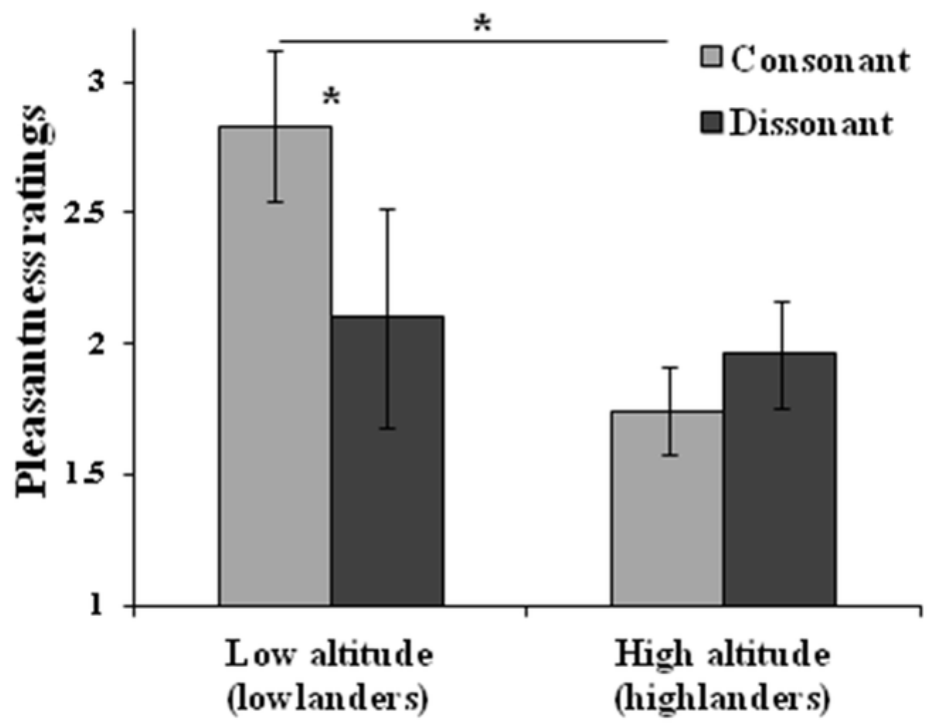

B)

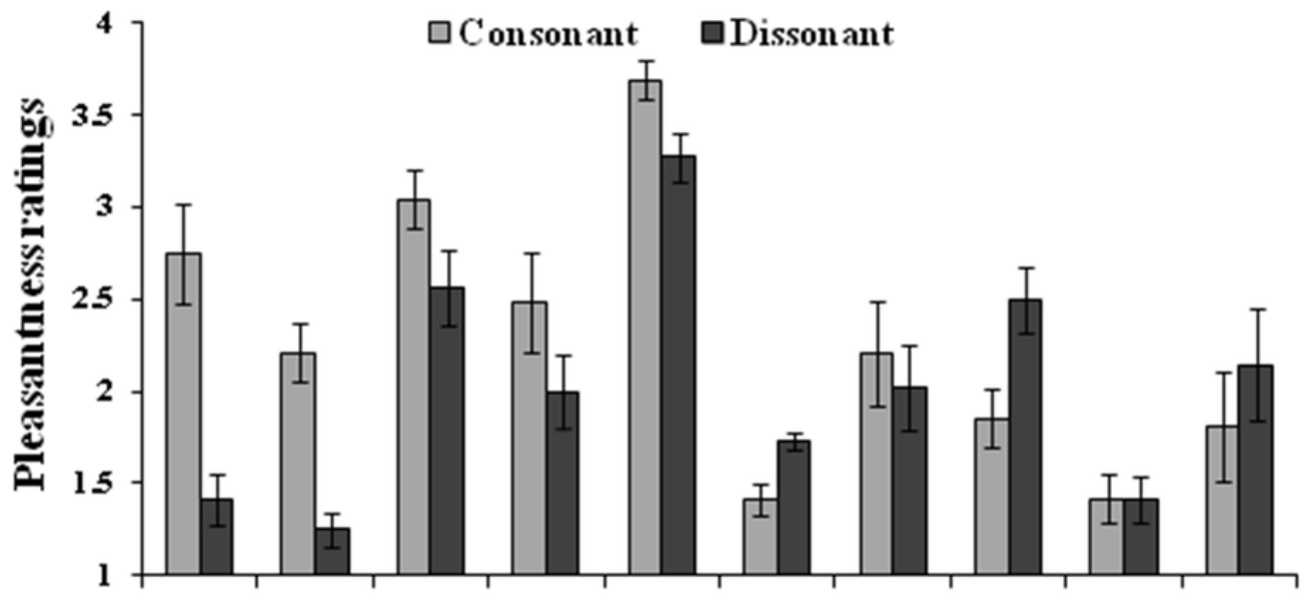

Low 1 Low 2 Low3 Low4 Low5 High1 High2 High3 High4 High5

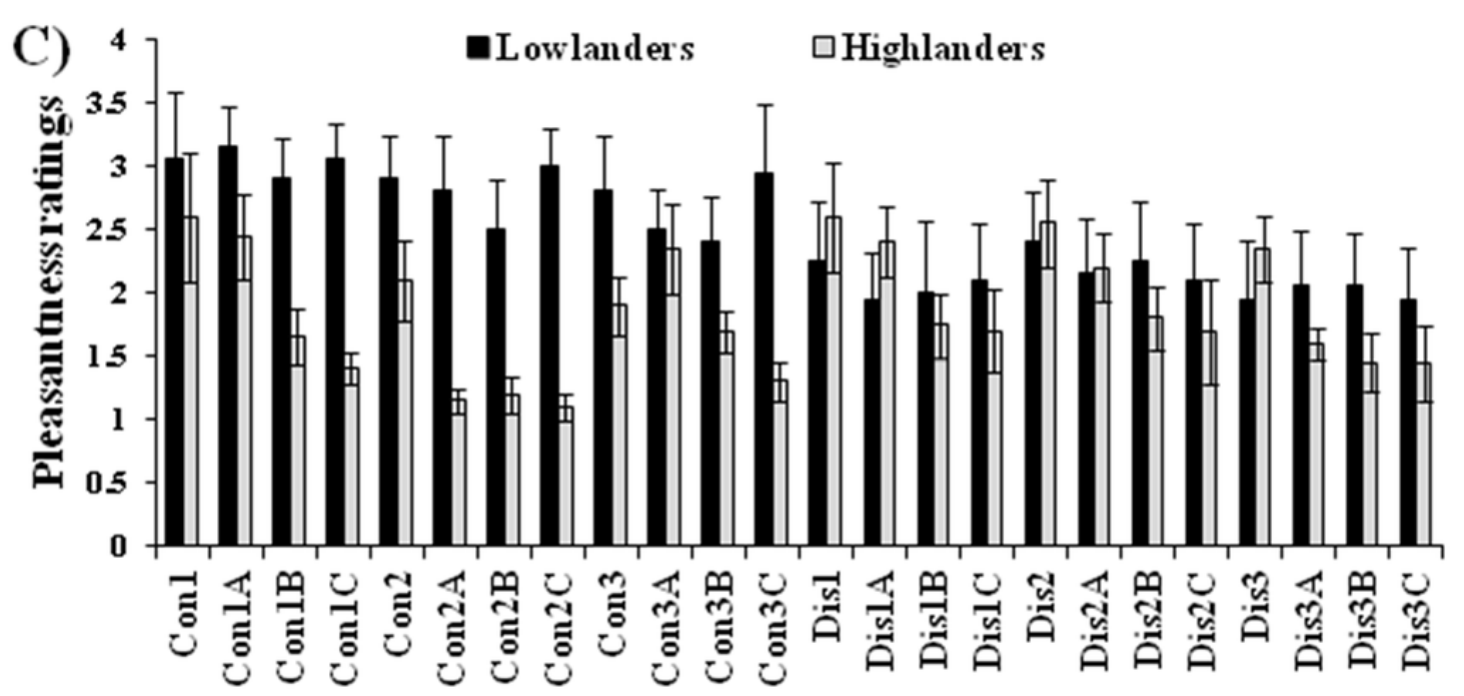

